\title{
Nanocomposite - From Improved Properties to Fundamental Understanding
}

Qing $\mathrm{Hao}^{*}$

DOI: $10.30919 / \mathrm{esmm} 5 \mathrm{f} 186$

Nanocomposites have been widely studied for their dramatically improved properties, such as mechanical strength, thermoelectric performance, chemical reactivity, and optical properties. With new materials and nanomanufacturing techniques, new opportunities are further introduced to this long-running research direction. This issues have multiple interesting papers dedicated to this important topic.

For mechanical properties, Hu et al. (DOI: 10.30919/esmm5f158) demonstrated the use of hydroxide $\left(\mathrm{Ca}(\mathrm{OH})_{2}\right)$ nano-spherulites (CNS) with $<5 \mathrm{~nm}$ diameters as cross-linkers to achieve highly stretchable selfhealing nanocomposite hydrogel. For solid lubricant applications, Joseph et al. (DOI: 10.30919/esmm5f152) added $\mathrm{MoS}_{2}$ nanosheets to plated Ni coatings to dramatically improve the microhardness and wear resistance of the obtained Ni-MoS $\mathrm{M}_{2}$ composite.

Improvements can also be found on the chemistry side. By coating ultrathin $\mathrm{MoSe}_{2}$ nanosheets onto hollow carbon spheres (HCS), Yuan et al. (DOI: 10.30919/esmm5f112) reported remarkable hydrogen evolution reaction activity, which was attributed to more active edges and the beneficial conductivity provided by the HCS core. Guo et al. (DOI: $10.30919 /$ esmm5f168) reported $\mathrm{TiO}_{2} / \beta$-cyclodextrin organicinorganic hybrid nano-material with outstanding photocatalytic activity and recyclability on photocatalytic water splitting. In addition, Wei et al. (DOI: 10.30919/esmm5f162) showed cross-linked porous corn starch with high methyl violet adsorption capability. As a review, Wang et al. (DOI: 10.30919/esmm5f175) discussed the current status of nanocomposite adsorbents to remove heavy metal from wastewater.

In addition to the property improvement, "side effects" of some nanostructure additives should also be addressed. For example, Ghahari (DOI: 10.30919/esmm5f172) studied the elongated induction period time and delayed hydration process for cement pastes due to added ZnO-based nanoparticles.

On the fundamental side, Magnone et al. (DOI: 10.30919/esmm5f176) proposed some explanations for the point of discontinuity on the Arrhenius relationship for the $\mathrm{Ba}_{0.5} \mathrm{Sr}_{0.5} \mathrm{Co}_{1-x} \mathrm{Fe}_{\mathrm{x}} \mathrm{O}_{3-\delta}$ solid solution. In their argument, the commonly used Arrhenius law with a single activation energy is not applicable to such complicated solid solutions involving multiple chemical/physical processes. Based on simulations, Zhao et al. (DOI: 10.30919/esmm5f135) showed that the growth of Ni-Cu alloy dendrite was affected by the interface thickness. This can provide important guidance for such structure growth.

In summary, there exists many practical and fundamental problems for the future studies of nanocomposite and generally nanostructured materials. A better understanding of these problem can pave the way for the advancement of many mechanical- and chemical-related applications. We anticipate to see more papers in coming issues and welcome new efforts for other directions, such as thermal and optical properties of nanostructured materials and their large-scale manufacturing processes. 